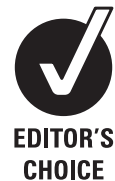

doi: 10.1136/ebm1030

Julie Solomon

Publisher, BMJ Publishing Group

Ltd, BMA House, Tavistock

Square, London WC1H 9JR, UK

\title{
New look Evidence-Based Medicine
}

\section{Julie Solomon}

In this issue of Evidence-Based Medicine, you will notice several differences. We have changed not only the visual appearance, but also the content and the type of articles. This is in response to feedback we have received over the past few years and the changing landscape of medical publishing. By initiating these changes, we plan to address two main concerns: timeliness and relevance to daily work.

More information than ever before is now available electronically, and more subscribers access the journal online than in print, but Evidence-Based Medicine has not fully reacted to these changes. The delay between the original articles being published and the value-added content appearing in Evidence-Based Medicine has become unacceptably long. This delay is attributable partly to the time taken by the filtering, writing, commissioning and editing process and partly to the journal's content being batched for publication on a bimonthly schedule. In order to address this problem, the journal will, over the coming year, switch from a batched publication model to a continuous publication model. This means that articles will be published on the website http://ebm.bmj.com as they become ready, removing months from the publication schedule. Another element of the delay was attributable to the abstracting process, as abstracts were being rewritten with evidence methods in mind. Feedback received, however, indicated that the specialist knowledge required to create these rewritten abstracts was not the focus of most readers' attention. Readers will notice, therefore, that the selected articles no longer consist of an abstract and a commentary. Instead, the citation for the original article being discussed will be included as a link so that interested readers can access the original abstract, directly, themselves. The extra space available as a result of not including the abstract will enable the commentaries to be more fully developed and to explain in more detail the why and the how of the articles, as well as their implications for daily work.

Evidence-Based Medicine has been more akin to a database than a journal. One way to improve its readability is to vary the type of material being included, not just in terms of format but also in terms of its organisation, orientation and visual format. In this issue you will find content sourced from another BMJ Group publication, Drug and Therapeutics Bulletin. This monthly publication provides an independent view of medical treatment and publishes "clear, succinct information and practical advice on medicines, other treatments and the overall management of disease'. We hope you will like the taster included here.

In the information age, we should be thinking how to make the content relevant to a broader, less specialised audience, and one that may not be as focused on the methods used but on the meaning of the outcome. We should be focusing on reaching out not just to doctors, nurses and academics but also to the reading public. Over the coming months, we will look forward to receiving feedback from you on the new approach and suggestions for content to include. Feel free to get involved; many hands will make light work of building the evidence base. 